\title{
Interaction Between Oblique Waves and Multiple Bottom-Standing Flexible Porous Barriers near a Rigid Wall
}

\author{
H. Behera ${ }^{\mathrm{a}, *}$, Chiu-On Ng ${ }^{\mathrm{b}}$ \\ ${ }^{a}$ SRM Research Institute and Department of Mathematics, SRM University, Kattankulathur, Tamil Nadu, India \\ ${ }^{b}$ Department of Mechanical Engineering, The University of Hong Kong, Pokfulam Road, Hong Kong, China
}

\begin{abstract}
The interaction between oblique surface waves and multiple bottom-standing flexible porous barriers under the combined effects of a neighboring rigid vertical wall and a step of arbitrary profile on the bottom is investigated. The problem is analyzed under the assumptions of small-amplitude water waves and structural response. The solutions are found using the methods of least-squares approximation, eigenfunction expansion and multi-mode approximation associated with the modified mild-slope equation. To keep the barriers at a desired position of interest, clamped-free or clamped-moored edge conditions are considered. Effects of various wave and structural parameters are studied for single, double and multiple barriers by looking into the reflection coefficient, wave force exerted on the rigid wall, free-surface elevations, and plate deflection of the barriers. The model is validated by comparing with results available in the literature for the special case of wave interaction with single and double rigid porous barriers near a rigid wall in the presence of a vertical step. The study reveals that the presence of multiple flexible porous barriers may effectively reduce the wave reflection and wave force exerted on the rigid wall. Further, full and nearly zero wave reflection can be found in the case of single and/or multiple barriers.
\end{abstract}

Keywords: Wave reflection; bottom-standing flexible porous barriers; mild-slope approximation; least-squares approximation.

\section{Introduction}

Breakwaters are barriers built in a body of water to protect a coast from the effect of waves. The design of a breakwater nowadays needs to take into account environmental impact of the structure. To be environmentally feasible, an innovative design is often required. The performance of a breakwater in defending a coast against wave attack depends on, among other factors, the hydrographic and wave conditions of the site. The wave-structure interaction problem is usually so complicated that the wave transformation on passing through the structure, or the wave loading on the structure, cannot be evaluated using simple formulas or standard design charts. To solve the problem, one may resort to mathematical modeling, analytical or numerical, which can be easily adapted to suit different conditions.

\footnotetext{
${ }^{*}$ Corresponding Author

Email addresses: hkb.math@gmail . com (H. Behera), cong@hku.hk (Chiu-On Ng)
} 
Among many other types, perforated or porous breakwaters have been increasingly introduced into the art of dissipating wave energy in nearshore regions as to provide safe areas for ship berthing. As early as Jarlan [1], a perforated wall breakwater was proposed to consist of a front porous wall, a rigid back wall and a wave absorbing chamber between them. Since then, Jarlan-type breakwater has been receiving considerable attention for its tested effectiveness on the reduction of wave reflection and wave force. Suh and Park [2] developed an analytical model using the Galerkin-eigenfunction method to predict the reflection coefficient of a perforated wall caisson mounted on a rubble mound foundation when waves are obliquely incident to the breakwater at an arbitrary angle. Sahoo et al. [3] studied wave reflection by a partial porous barrier of various geometries and configurations near a rigid wall using the least-squares approximation method. They found that when the distance between the porous barrier and the rigid wall is equal to a quarter wave length plus an integer multiple of half the wavelength of the incident wave, the wave reflection attains a minimum. The interaction of oblique waves with double-layered perforated breakwater was investigated by Li et al. [4] using the eigenfunction expansion method. Teng et al. [5] developed an analytic solution based on domain decomposition for oblique waves interacting with an infinite number of perforated caissons. Liu et al. [6] then presented a model for assessing the reflection of oblique waves from an infinite array of partially perforated caissons. This was followed by Liu et al. [7], who considered a modified type of perforated breakwater consisting of a porous front wall, a rigid back wall and a horizontal porous plate in the chamber. A thorough review of the developments on wave interaction with various perforated breakwater can be found in [8] and the literature cited therein. Behera et al. [9] studied wave trapping by a thin porous barrier near a rigid wall in the presence of a bottom step of arbitrary profile. Recently, Liu et al. [10] looked into perforated caisson breakwaters having perforated partition walls. Being light in weight, economical, reusable and environmental friendly, vertical fully/partial flexible porous barriers are preferred in many situations as a perforated breakwater. Yip et al. [11] studied wave trapping by a partial flexible porous plate of various configurations near a rigid wall. Recently, Behera et al. [12] extended the problem to wave trapping by a flexible porous barrier near a rigid wall in two-layer fluid. Kaligatla et al. [13] further studied the efficiency of a partial flexible porous barrier using free-surface Green's function.

By various mathematical techniques, wave interactions with fully/partial single/multiple barriers without a rigid back wall have been extensively investigated in recent decades. Wave scattering by a surface-piercing vertical flexible sheet was studied by Meylan [14] using Fredholm-type integral equations and Green's function. Mandal and Chakrabarti [15] reviewed various methods for wave scattering by partial rigid barriers. Lee and Lo [16] analyzed the performance of both single and dual surface-piercing membrane barrier systems. Karmakar et al. [17] developed a model for wave scattering by multiple bottom-standing flexible porous barriers using the methods of least squares and wide-spacing approximation. Several aspects of wave scattering by multiple surface-piercing floating membrane were studied by Karmakar and Soares [18], who reported that the number of barriers and the barrier spacing can play a central role in controlling wave transmission. Apart from wave interaction with vertical structures, there has been significant progress on wave past horizontal structures of different configurations. Hassan et al. [19] developed an analytic solution using eigenfunc- 
tion method for wave scattering by submerged elastic plate. Meylan et al. [20] used eigenfunction, boundary-element and finite-element method for wave interaction with floating porous elastic plate. Das and Sahoo [21] developed a solution for wave interaction with very large floating elastic plate over viscoelastic bed. Recently, Koley and Sahoo [22] studied oblique wave scattering by horizontal floating flexible porous membrane using Green's function technique.

Although wave interaction between porous barriers in the case of a flat bottom has been well studied in the literature, few of the existing work has considered the simultaneous effects due to a neighboring rigid wall and a stepped bottom. This has formed the motivation of the present study, which aims to look into wave interaction with multiple bottom-standing flexible porous barriers subject to the combined effects of a rigid side wall and a step of arbitrary profile on the bottom. In the present study, modified mild-slope equation (MMSE) of Chamberlain and Porter [23] is solved by using the Runge-Kutta for determining velocity potentials in the fluid domain having bottom undulation while the eigenfunction expansion method is used for velocity potentials in the fluid domain having a uniform water depth, and the solution of the MMSE is matched at the bottom slope discontinuities through mass conserving jump conditions derived by Porter and Staziker [24]. Moreover, the leastsquares approximation method is used to find the solutions of the physical problem in the presence of multiple bottom-standing partial flexible porous barriers. Clamped-free or clamped-moored edge conditions are used to describe the fixing of barriers at a desired position of interest. Numerical results are shown to reveal the effects of various wave and structural parameters on the reflection coefficient, wave force on the rigid wall, free-surface elevations, and deflection of the barriers. The computed results are compared with those available in the literature for the special case of wave interaction in the presence of a vertical step.

\section{Mathematical Formulation}

Oblique wave interaction with multiple bottom-standing flexible porous barriers is studied in the presence of a step-type bottom of arbitrary profile under the assumptions of linearized water wave theory and small-amplitude structural response. The problem is considered in the three-dimensional Cartesian co-ordinate system with $x$ - $y$ being the horizontal plane and the $z$-axis being vertically upward. It is considered that the step occupies the region $0<x<L$ with variable depth $h(x)$, and a uniform open water region $-\infty<x<0$ and a coastal region $L<x<L_{1}+\cdots+D$ with water depths $h_{1}$ and $h_{2}$, respectively. It is assumed that the $N$ bottom-standing barriers are equally spaced and placed at $x=\alpha_{j}$, where $\alpha_{j}=L+L_{1}+(j-3) L_{2}$ for $j=3,4, \cdots,(N+2)$. The first barrier is located at a distance $L_{1}$ from the end edge of the step, $B$ is the distance between the first barrier and the rigid wall, $L_{2}$ is the spacing between adjacent barriers, and $D$ is the distance between the last barrier and the rigid wall, as shown in Fig. 1. It is assumed that the fluid is inviscid, incompressible, and the motion is irrotational and simple harmonic in time with angular frequency $\omega$. The body of fluid is assumed to extend horizontally along the $y$-axis over $-\infty<y<\infty$. Obliquely incident waves over a sloping bed is a quasi-3D problem. If the bottom changes only in the $x$-direction, the wave component does not change in its wave number in the $y$-direction. Thus, the form of the velocity potential for $j=1,2,3, \cdots,(N+3)$ is given by $\Phi_{j}(x, y, z, t)=\operatorname{Re}\left\{\phi_{j}(x, z) \mathrm{e}^{-\mathrm{i}\left(k_{y} y+\omega t\right)}\right\}$, where $\theta$ is the incident angle 
with respect to the $x$-axis and $k_{y}=k_{10} \sin \theta$ with $k_{10}$ being the wave number of the incident wave in region 1. Along the vertical $z$-direction, $H_{b} \in\left(-h_{2},-h_{2}+b\right)$ and $H_{g} \in\left(-h_{2}+b, 0\right)$ denote the barrier and gap regions, respectively, with $b$ being the length of the barriers.

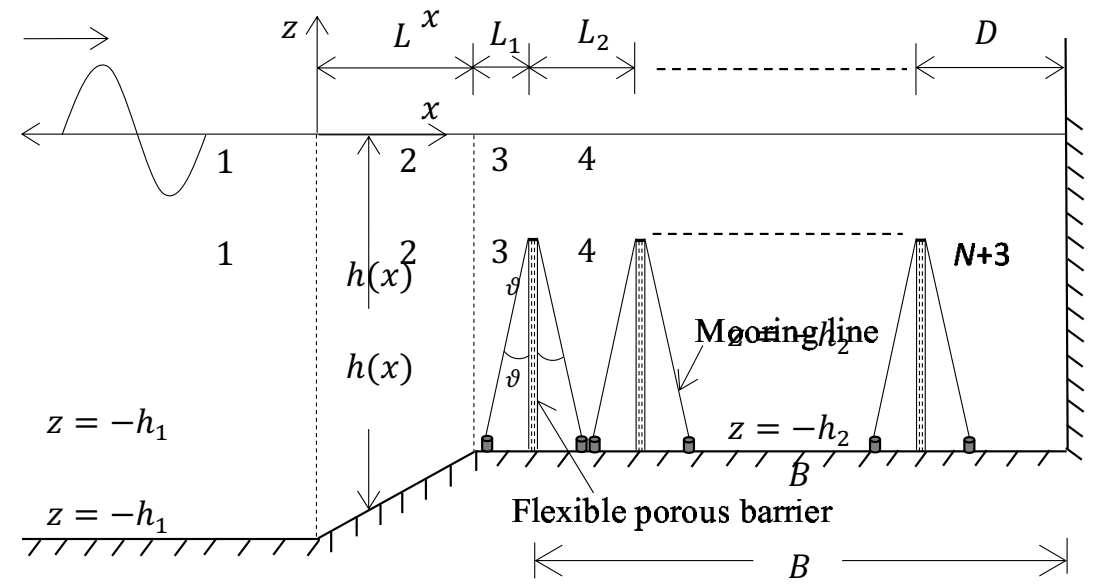

(a) Side view

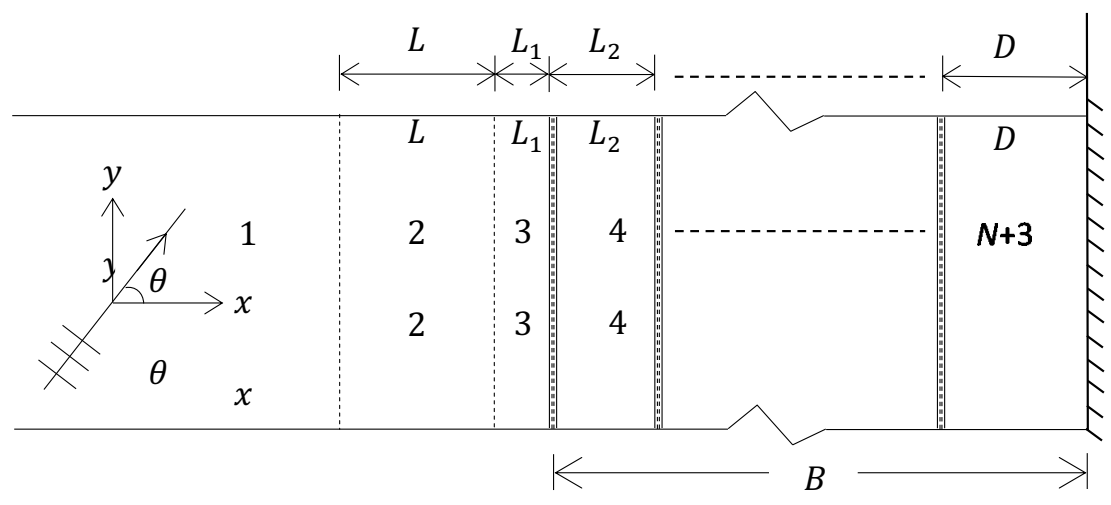

(b) Top view

Figure 1: Wave interaction with multiple bottom-standing flexible porous barriers near a rigid wall.

The spatial velocity potential $\phi_{j}(x, z)$ for $j=1,2,3, \cdots,(N+3)$ satisfies the Helmholtz equation given by

$$
\left(\nabla_{x z}^{2}-k_{y}^{2}\right) \phi_{j}=0
$$

where $\nabla_{x z}^{2}=\left(\partial^{2} / \partial x^{2}+\partial^{2} / \partial z^{2}\right)$. The linearized free-surface boundary condition is given by

$$
\frac{\partial \phi_{j}}{\partial z}-K \phi_{j}=0 \quad \text { on } z=0, \text { for } j=1,2, \cdots,(N+3),
$$

where $K=\omega^{2} / g$, and $g$ is the acceleration due to gravity. Further, the boundary condition on the two horizontal bottoms is given by

$$
\frac{\partial \phi_{j}}{\partial z}=0 \quad \text { on } \quad z=-h_{i},
$$


where $i=1$ for $j=1$, while $i=2$ for $j=3,4, \cdots,(N+3)$. On the other hand, the bottom boundary condition for the step region 2 on $z=-h(x)$ is given by

$$
\frac{\partial \phi_{2}}{\partial z}+\frac{d h}{d x} \frac{\partial \phi_{2}}{\partial x}=0
$$

The far-field boundary conditions is given by

$$
\phi_{1}=\left(A_{10} \mathrm{e}^{\mathrm{i} p_{10} x}+B_{10} \mathrm{e}^{-\mathrm{i} p_{10} x}\right) f_{10}\left(k_{10}, z\right), \text { as } x \rightarrow-\infty
$$

where $A_{10}$ is the incident wave amplitude, and $B_{10}$ is the complex amplitude of the reflected wave. Further, $k_{10}$ is the real root of the dispersion relation in region 1 with $p_{10}=\sqrt{k_{10}^{2}-k_{y}^{2}}$, and $f_{10}\left(k_{10}, z\right)$ being the associated vertical eigenfunctions. On the impermeable end-wall, the vanishing of the horizontal velocity gives

$$
\frac{\partial \phi_{(N+3)}}{\partial x}=0 \quad \text { on } \quad x=\alpha_{(N+3)} .
$$

The continuity of pressure and normal velocity along the gap at $x=\alpha_{j}$ for $j=3,4, \cdots,(N+2)$ are given by

$$
\phi_{j}=\phi_{j+1} \quad \text { and } \quad \frac{\partial \phi_{j}}{\partial x}=\frac{\partial \phi_{j+1}}{\partial x}, \quad z \in H_{g} .
$$

The flexible barriers are assumed to be oscillating in the horizontal direction with displacement of the form $\zeta_{(j-2)}(y, z, t)=\operatorname{Re}\left\{\xi_{(j-2)}(z) e^{-\mathrm{i}\left(k_{y} y-\omega t\right)}\right\}$, where $\xi_{(j-2)}(z)$ for $j=3,4, \cdots,(N+2)$ are the complex deflection amplitudes of the flexible barriers. Thus, the boundary condition on the flexible porous barriers at $x=\alpha_{j}$ for $j=3,4, \cdots,(N+2)$ based on Darcy's law is given by

$$
\frac{\partial \phi_{j}}{\partial x}=\mathrm{i} k_{10} G\left(\phi_{j}-\phi_{j+1}\right)-\mathrm{i} \omega \xi_{(j-2)}, \quad z \in H_{b},
$$

where $G$, given below, is the complex porous-effect parameter as defined by Yu and Chwang [25]:

$$
G=G_{r}+\mathrm{i} G_{i}=\frac{\epsilon(f+\mathrm{i} S)}{k_{10} d_{s}\left(f^{2}+S^{2}\right)}
$$

with $\epsilon$ the porosity of the barrier, $f$ the resistance force coefficient, $S$ the inertial force coefficient, and $d_{s}$ the thickness of the porous barrier. Further, the real part $G_{r}$ represents the resistance effect of the porous material against the seepage flow while the imaginary part $G_{i}$ denotes the inertia effect of the fluid inside the porous material. The equation of motion of the barrier at $x=\alpha_{j}$ for $j=3,4, \cdots,(N+2)$ acted upon by fluid pressure yields (as in Magrab [26])

$$
E I\left(\frac{d^{2}}{d z^{2}}-k_{y}^{2}\right)^{2} \xi_{(j-2)}+Q\left(\frac{d^{2}}{d z^{2}}-k_{y}^{2}\right) \xi_{(j-2)}-m_{s} \omega^{2} \xi_{(j-2)}=\mathrm{i} \rho \omega\left(\phi_{j}-\phi_{j+1}\right), \quad z \in H_{b},
$$

where $E I=E d_{s}^{3} / 12\left(1-v^{2}\right)$ is the rigidity of the barriers, $E$ is the Young's modulus, $d_{s}$ is the thickness of the barrier, $v$ is the Poisson's ratio, $Q$ is the uniform compressive force acting on the barrier, and $m_{s}=\rho_{s} d_{s}$ is the uniform mass per unit length with $\rho_{s}$ being the barrier density. For a unique solution of the boundary value problem and to keep the flexible barrier in position, the partial barriers are 
subjected to certain edge conditions at the two ends of the barrier. In the present study, two possible types of edge conditions are considered as follows.

(i) Clamped-free: The barrier is assumed to be clamped near the sea bed at $\left(\alpha_{j},-h_{2}\right)$ and free near the submerged end at $\left(\alpha_{j},-h_{2}+b\right)$ for $j=3,4, \cdots,(N+2)$. In the case of a clamped edge, the barrier deflection and the slope of the barrier deflection are zero, while near the free edge, the bending moment and shear force are zero. Thus, the edge conditions are given by

$$
\left.\begin{array}{l}
\xi_{(j-2)}(u)=0, \xi_{(j-2)}^{\prime}(u)=0, \\
\left(\frac{d^{2}}{d z^{2}}-v k_{y}^{2}\right) \xi_{(j-2)}(u)=0,\left[E I\left\{\frac{d^{2}}{d z^{2}}-(2-v) k_{y}^{2}\right\} \frac{d}{d z}+Q \frac{d}{d z}\right] \xi_{(j-2)}(u)=0 .
\end{array}\right\}
$$

(ii) Clamped-moored: The barrier is assumed to be clamped near the sea bed at $\left(\alpha_{j},-h_{2}\right)$ and moored near the submerged end at $\left(\alpha_{j},-h_{2}+b\right)$ for $j=3,4, \cdots,(N+2)$. In the case of a moored edge, the bending moment is zero and the horizontal components of the dynamic mooring line tensions are related to the restoring forces due to the axial load to the shearing forces as given by:

$$
\left.\begin{array}{l}
\xi_{(j-2)}(u)=0, \xi_{(j-2)}^{\prime}(u)=0, \\
\left(\frac{d^{2}}{d z^{2}}-v k_{y}^{2}\right) \xi_{(j-2)}(u)=0,\left[E I\left\{\frac{d^{2}}{d z^{2}}-(2-v) k_{y}^{2}\right\} \frac{d}{d z}+Q \frac{d}{d z}\right] \xi_{(j-2)}(u)=\left(2 K_{m} \sin ^{2} \vartheta\right) \xi_{(j-2)}(u),
\end{array}\right\}
$$

where $K_{m}$ is the mooring line stiffness, $\vartheta$ is the mooring line angle, and $u$ is $-h_{2}+b$ or $-h_{2}$ as appropriate.

\section{Method of Solution}

In this section, the method of solution for the present problem is briefly discussed. The methods of least-squares approximation and multi-mode approximation associated with the modified mild slope equation are used to determine the unknown coefficients associated with the velocity potentials in each region. Using the expansion formulas, the form of the special velocity potentials $\phi_{j}(x, z)$ for $j=1,2, \cdots,(N+3)$ in each $(\mathrm{N}+3)$ regions are expressed as

$$
\phi_{j}(x, z)= \begin{cases}A_{10} e^{\mathrm{i} p_{10} x} f_{10}\left(k_{10}, z\right)+\sum_{n=0}^{\infty} B_{1 n} e^{-\mathrm{i} p_{1 n} x} f_{1 n}\left(k_{1 n}, z\right), & x<\left(\alpha_{1}=0\right), j=1, \\ \sum_{n=0}^{\infty} \psi_{n}(x) W_{n}(h(x), z), & \alpha_{1}<x<\left(\alpha_{2}=L\right), j=2, \\ \sum_{n=0}^{\infty}\left(A_{j n} e^{\mathrm{i} p_{2 n} x}+B_{j n} e^{-\mathrm{i} p_{2 n} x}\right) f_{2 n}\left(k_{2 n}, z\right), & \alpha_{(j-1)}<x<\alpha_{j}, j=3,4, \cdots,(N+2), \\ \sum_{n=0}^{\infty} B_{j n} \cos p_{2 n}\left(x-\alpha_{(N+3)}\right) f_{2 n}\left(k_{2 n}, z\right), & \alpha_{(N+2)}<x<\alpha_{(N+3)}, j=N+3,\end{cases}
$$

where $\alpha_{(N+3)}=\alpha_{(N+2)}+D, f_{i n}\left(k_{i n}, z\right)=\cosh k_{i n}\left(z+h_{i}\right) / \cosh k_{i n} h_{i}, p_{i n}=\sqrt{k_{i n}^{2}-k_{y}^{2}}$ for $i=1,2$, in which $k_{10}$ and $k_{20}$ are the positive real roots and $k_{\text {in }}$ for $n=1,2,3, \cdots$ are the purely imaginary roots of the dispersion equation $\omega^{2}=g k_{\text {in }} \tanh k_{i n} h_{i}$ in $k_{i n}$ for $i=1,2$. Further, in the step region, $\psi_{n}(x)$ 's are unknown functions and $W_{n}=\cosh \tilde{k}_{n}(z+h) / \cosh \tilde{k}_{n} h$, where the wave number $\tilde{k}_{0}$ is a positive real root and $\tilde{k}_{1}, \tilde{k}_{2}, \tilde{k}_{3}, \cdots$ are purely imaginary roots of the dispersion equation $\omega^{2}=g \tilde{k} \tanh \tilde{k} h$ in $\tilde{k}$. One 
should note that the roots $\tilde{k_{0}}, \tilde{k_{1}}, \tilde{k_{2}}, \tilde{k_{3}}, \cdots$ are functions of the bottom profile $h(x) . A_{j n}$ and $B_{j n}$ are the unknown constants to be determined. Hereafter, the infinite series associated with the evanescent modes for the velocity potentials are truncated to $M$ terms. Using the same procedure as in [24] for extended modified mild-slope equation to find $\psi_{n}(x)$ in the undulated region, it can be easily derived that

$$
\frac{d}{d x}\left(a_{n} \frac{d \psi_{n}}{d x}\right)+\sum_{m=0}^{M}\left[\left(b_{m n}-b_{n m}\right) \frac{d h}{d x} \frac{d \psi_{m}}{d x}+\left\{b_{m n} \frac{d^{2} h}{d x^{2}}+c_{m n}\left(\frac{d h}{d x}\right)^{2}+d_{m n}-k_{y}^{2} a_{n}\right\} \psi_{m}\right]=0,
$$

where

$$
\begin{aligned}
& a_{n}(h)=\int_{-h}^{0} W_{n}^{2} d z, \quad b_{m n}(h)=\int_{-h}^{0} W_{n} \frac{\partial W_{m}}{\partial h} d z, \\
& c_{m n}(h)=\frac{d b_{m n}}{d h}-\int_{-h}^{0} \frac{\partial W_{m}}{\partial h} \frac{\partial W_{n}}{\partial h} d z, d_{m n}(h)=\int_{-h}^{0} W_{n} \frac{\partial^{2} W_{m}}{\partial z^{2}} d z,
\end{aligned}
$$

for $n=0,1,2, \cdots, M$. Using the velocity potential as in Eq. (13) and the continuity of pressure across the interfaces $x=0$ and $x=L$, we get

$$
\begin{aligned}
& \left.\begin{array}{l}
\psi_{0}(x)=A_{10} e^{\mathrm{i} p_{10} x}+B_{10} e^{-\mathrm{i} p_{10} x}, \\
\psi_{n}(x)=B_{1 n} e^{-\mathrm{i} p_{1 n} x},
\end{array}\right\} \text { at } x=0 \text { for } n=1,2, \cdots, M, \\
& \psi_{n}(x)=A_{3 n} e^{\mathrm{i} p_{2 n} x}+B_{3 n} e^{-\mathrm{i} p_{2 n} x} \text { at } x=L \text { for } n=0,1,2, \cdots, M .
\end{aligned}
$$

Using Eqs. (15) and (16) and the conservation of mass across the interface boundaries at $x=0$ and $L$, as in [24], we get the jump conditions as follows:

$$
\left.\begin{array}{c}
a_{0} \frac{d \psi_{0}}{d x}+\mathrm{i} p_{10} a_{0} \psi_{0}+h^{\prime} \sum_{m=0}^{M} b_{m 0} \psi_{m}-2 \mathrm{i} p_{10} a_{0} A_{10}=0, \\
a_{n} \frac{d \psi_{n}}{d x}+\mathrm{i} p_{1 n} a_{n} \psi_{n}+h^{\prime} \sum_{m=0}^{M} b_{m n} \psi_{m}=0,
\end{array}\right\} \text { at } x=0+, n=1,2, \cdots, M,
$$

Using the velocity potentials $\phi_{j}$ for $j=3,4, \cdots,(N+3)$ as in Eq. (13) and the continuity of velocity at $x=\alpha_{j}$ as in Eq. (7) along with the orthogonal characteristics of the eigenfunctions $f_{2 n}\left(k_{2 n}, z\right)$ for $n=0,1,2, \cdots$, we get

$$
\left.\begin{array}{l}
A_{j n}-B_{j n} e^{-2 \mathrm{i} p_{2 n} \alpha_{j}}=A_{(j+1) n}-B_{(j+1) n} e^{-2 \mathrm{i} p_{2 n} \alpha_{j}}, \text { for } j=3,4, \cdots,(N+1), \\
A_{j n} e^{\mathrm{i} p_{2 n} \alpha_{j}}-B_{j n} e^{-\mathrm{i} p_{2 n} \alpha_{j}}=-\mathrm{i} B_{(j+1) n} \sin \left(p_{2 n} D\right), \text { for } j=(N+2) .
\end{array}\right\}
$$

Using Eqs. (13) and (19) in Eq. (10), the deflection of the flexible barriers $\xi_{(j-2)}(z)$ at $x=\alpha_{j}$ for $j=3,4, \cdots,(N+2)$ is obtained as follows:

$$
\xi_{(j-2)}(z)=\sum_{m=1}^{4} C_{j m} g_{m}(z)+\sum_{n=0}^{M} E_{n}\left(U_{j n} B_{j n}-V_{j n} B_{(j+1) n}\right) f_{2 n}\left(k_{2 n}, z\right), \quad z \in H_{b},
$$


where $U_{j n}=2 e^{-\mathrm{i} q_{2 n} \alpha_{j}}$ and $C_{j m}$ for $j=3,4, \cdots,(N+2)$ are the unknown constants with $\tau_{n}$ 's being the roots of the characteristic equation $E I\left(\tau_{n}^{2}-k_{y}^{2}\right)^{2}+Q\left(\tau_{n}^{2}-k_{y}^{2}\right)-m_{s} \omega^{2}=0$ and $\tau_{n}=\mathrm{i} \tau_{n}$ for $n=3,4$. Further, in Eq. (20)

$$
\begin{aligned}
& V_{j n}=\left\{\begin{array}{ll}
2 e^{-\mathrm{i} q_{2 n} \alpha_{j}}, & j=3,4, \cdots,(N+1), \\
s_{n}, & j=N+2,
\end{array} \quad \begin{array}{l}
E_{n}=\frac{\mathrm{i} \rho \omega}{E I p_{2 n}^{4}+Q p_{2 n}^{2}-m_{s} \omega^{2}}, \\
g_{1}(z)=\frac{\cosh \tau_{1} z}{\cosh \tau_{1} h_{2}}, g_{2}(z)=\frac{\sinh \tau_{2} z}{\sinh \tau_{2} h_{2}}, g_{3}(z)=\frac{\cos \tau_{3} z}{\cos \tau_{3} h_{2}}, g_{4}(z)=\frac{\sin \tau_{4} z}{\sin \tau_{4} h_{2}},
\end{array}\right.
\end{aligned}
$$

where $s_{n}=\mathrm{i} \sin \left(p_{2 n} D\right)+\cos \left(p_{2 n} D\right)$. Using Eq. (19) and $\phi_{j}$ from Eq. (13) in Eq. (7) for $j=$ $3,4, \cdots,(N+2)$ at $\alpha_{j}$ yields

$$
\sum_{n=0}^{M}\left(U_{j n} B_{j n}-V_{j n} B_{(j+1) n}\right) f_{2 n}\left(k_{2 n}, z\right)=0, \quad \text { for } \quad z \in H_{g}
$$

Further, substituting $\phi_{j}$ and $\xi_{(j-2)}(z)$ for $j=3,4, \cdots,(N+2)$ at $x=\alpha_{j}$ from Eqs. (13) and (20) in Eq. (8), we obtain

$$
\sum_{n=0}^{M}\left(\tilde{a}_{j n} B_{j n}+\tilde{b}_{j n} A_{(j+1) n}+\tilde{c}_{j n} B_{(j+1) n}\right) f_{2 n}\left(k_{2 n}, z\right)+\mathrm{i} \omega \sum_{m=1}^{4} C_{j m} g_{m}(z)=0, z \in H_{b},
$$

where $\tilde{a}_{j n}=\mathrm{i} e_{n} U_{j n}$ for $j=3,4, \cdots,(N+2)$ with $e_{n}=\omega E_{n}-k_{10} G$, while $\tilde{b}_{j n}$ and $\tilde{c}_{j n}$ are given by $\tilde{b}_{j n}=\left\{\begin{array}{ll}\mathrm{i} e^{\mathrm{i} p_{2 n} \alpha_{j}}, & j=3,4, \cdots,(N+1), \\ 0, & j=N+2,\end{array} \tilde{c}_{j n}= \begin{cases}\mathrm{i} e_{n} V_{j n}, & j=3,4, \cdots,(N+1), \\ p_{2 n} \sin \left(p_{2 n} D\right)-\mathrm{i} e_{n} V_{j n}, & j=N+2 .\end{cases}\right.$

Now, using the least-squares approximation method, Eqs. (21) and (22) yield

$$
\int_{-h_{2}}^{0}\left|J_{M}(z)\right|^{2} d z=\text { minimum }
$$

where

$$
J_{M}(z)=\sum_{n=0}^{M}\left\{B_{j n} T_{j n}^{(1)}(z)+A_{(j+1) n} T_{j n}^{(2)}(z)+B_{(j+1) n} T_{j n}^{(3)}(z)\right\}+T_{j m}^{(4)}(z) \text { for } j=3,4, \cdots,(N+2),
$$

with

$$
\begin{aligned}
& T_{j n}^{(1)}=\left\{\begin{array}{ll}
\tilde{a}_{j n} f_{2 n}\left(k_{2 n}, z\right), & z \in H_{b}, \\
U_{j n} f_{2 n}\left(k_{2 n}, z\right), & z \in H_{g},
\end{array} T_{j n}^{(2)}= \begin{cases}\tilde{b}_{1 n} f_{2 n}\left(k_{2 n}, z\right), & z \in H_{b}, \\
0, & z \in H_{g},\end{cases} \right. \\
& T_{j n}^{(3)}=\left\{\begin{array}{ll}
\tilde{c}_{1 n} f_{2 n}\left(k_{2 n}, z\right), & z \in H_{b}, \\
V_{j n} f_{2 n}\left(k_{2 n}, z\right), & z \in H_{g},
\end{array} T_{j m}^{(4)}(z)= \begin{cases}\mathrm{i} \omega \sum_{m=1}^{4} C_{j m} \tilde{f}_{m}(z), & z \in H_{b}, \\
0, & z \in H_{g} .\end{cases} \right.
\end{aligned}
$$

Minimizing Eq. (24) with respect to $B_{(j+1) n}$, leads to

$$
\int_{-h_{2}}^{0} J_{M}^{*}(z) J_{B_{(j+1) n}}(z) d z=0,
$$


where $J_{B_{(j+1) n}}(z)$ is the derivative of $J_{M}(z)$ with respect to $B_{(j+1) n}$ and $*$ denotes the complex conjugate of $J_{M}(z)$. The substitution of Eq. (24) into Eq. (25) results in

$$
\sum_{m=0}^{M}\left(B_{j n}^{*} X_{m n}^{(1)}+A_{(j+1) n}^{*} X_{m n}^{(2)}+B_{(j+1) n}^{*} X_{m n}^{(3)}\right)=X_{m n}^{4}, \quad \text { for } j=3,4, \cdots,(N+2) \text { and } n=0,1, \cdots, M,
$$

with

$$
X_{m n}^{(i)}=\int_{-h_{2}}^{0} T_{j m}^{(i)^{*}}(z) T_{j n}^{(3)}(z) d z, \text { for } i=1,2,3,4
$$

Next, to find $\psi_{n}$, the modified mild-slope equation, given in Eq. (14), is solved using the Runge-Kutta method along with Eqs. (15)-(18) and the specified bed profile $h(x)$. Further, using the required edge conditions as in Eqs. (11) or (12), the computed results of $\psi_{n}$ and the system of equations in Eq. (26), $(6 N+4) \times M$ equations are solved for the unknown constants.

\section{Numerical Results and Discussion}

In this section, we discuss results generated by a MATLAB program that we have developed for investigating the effect of various wave and structural parameters on the wave reflection, hydrodynamic forces acting on the rigid back wall and plate deflections. In the present study, the number of barriers $N=4$, acceleration due to gravity $g=9.81 \mathrm{~m} / \mathrm{sec}^{2}, k_{10} h_{1}=1$, depth ratio $h_{2} / h_{1}=0.5$, porous-effect parameter $G=1, B / h_{1}=1$, spacing between the barrier $L_{2} / B=(1-D / B) /(N-1)$ for $N>1, L / h_{1}=0.2, D / B=0.4, \gamma=E I /\left(\rho g h_{2}^{4}\right)=0.1, \beta=Q /\left(\rho g h_{2}^{2}\right)=0, v=m_{s} /\left(\rho h_{2}\right)=0.1$, $v=0.3, b / h_{2}=1, \vartheta=45^{\circ}, K_{m}=10^{3} \mathrm{~N} \mathrm{~m}^{-1}$, and $\theta=40^{\circ}$ are considered unless stated otherwise. The reflection coefficient $K_{r}$ is defined as

$$
K_{r}=\frac{B_{10}}{A_{10}}
$$

Further, the non-dimensional form of the horizontal wave force exerted on the rigid wall is defined as $K_{w}=\left|C_{w}\right|$, where

$$
C_{w}=\left.\left(\frac{\mathrm{i} \omega}{g h_{2}^{2}}\right) \int_{-h_{2}}^{0} \phi_{(N+3)}(x, z)\right|_{x=\alpha_{(N+3)}} d z
$$

\subsection{Various bed profiles}

We consider the bed profiles shown in Figs. 2(a)-(d), which can be generated using the following bed function $h(x)$ :

$$
h(x)=h_{1}-\varrho\left\{1-\delta(1-x / L)^{2}+(\delta-1)(1-x / L)\right\}, \quad 0<x<L,
$$

where $\varrho=h_{1}-h_{2}$. Depending on the parameter $\delta$, Eq. (30) corresponds to various types of bed profile connecting the depths $h_{1}$ and $h_{2}$ : a plane sloping step for $\delta=0$ as in Fig. 2(a), a protrusion above the depth $h_{2}$ for $\delta>1$ as in Fig. 2(b), a concave profile for $-1 \leq \delta<0$ as in Fig. 2(c), and a depression below the depth $h_{1}$ for $\delta<-1$ as in Fig. 2(d) as in [27]. Unless stated otherwise, the value of $\delta=0$ is used. 


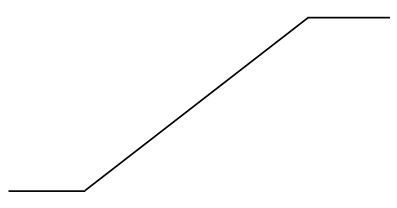

(a)

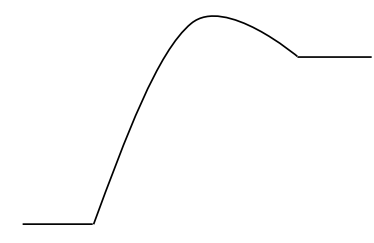

(b)

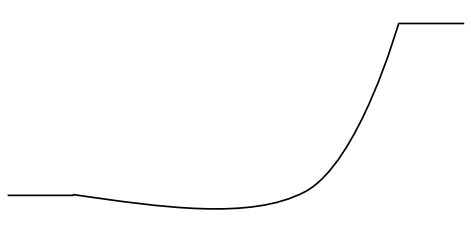

(c)

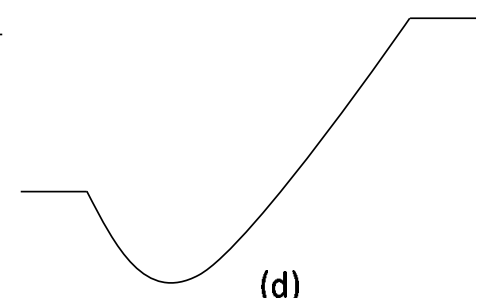

Figure 2: Schematic diagram of a set of bed profiles considered in the present study.

\subsection{Validation and comparison with other results}

In Figs. 3(a) and 3(b), the reflection coefficient versus the non-dimensional wave number $k_{10} h_{1}$ are plotted in the case of (a) rigid, and (b) flexible porous barriers for different numbers of barriers $N$. From Fig. 3(a), it is found that the curves of $K_{r}$ obtained by the present theory (for a steep step of $84.29^{\circ}$ ) are in close agreement with the results of Li et al. [4] for a vertical step. Li et al. [4] compared their numerical results with the experimental data. This comparison provides evidence on the accuracy of the results presented in this paper.

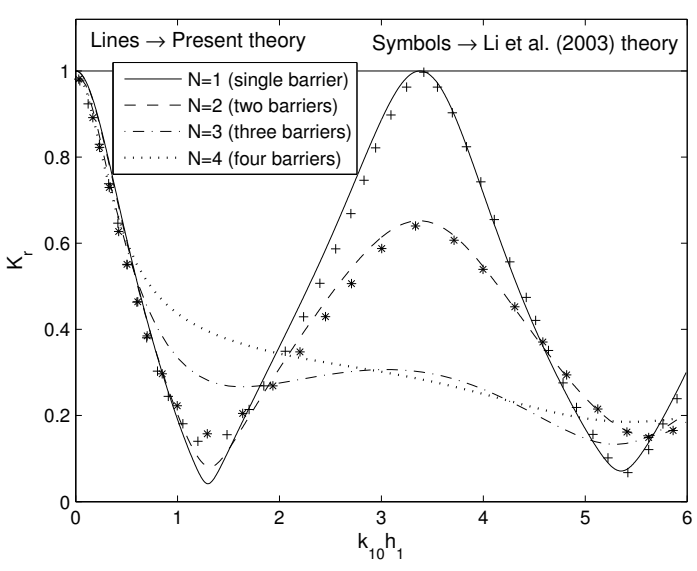

(a)

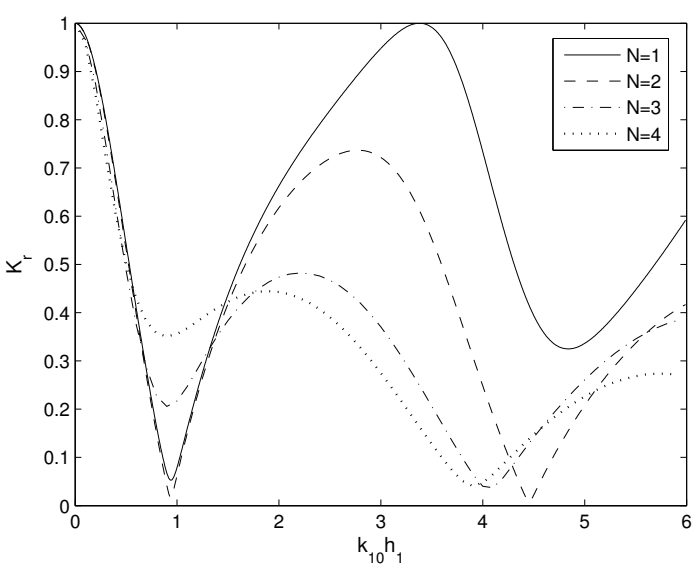

(b)

Figure 3: The reflection coefficients $K_{r}$ as a function of the non-dimensional incident wave number $k_{10} h_{1}$ for different numbers of barriers $N$ : (a) a comparison between present results with the results of Li et al. [4] with $\gamma=5$, and for (b) $\gamma=0.1, B / h_{1}=1, D / B=0.2, L / h_{1}=0.05, \beta=0, \vartheta=45^{\circ}, \theta=30^{\circ}, G=1, b / h_{2}=1$, and $h_{2} / h_{1}=0.5$.

From these figures, it is observed that full reflection does not occur for $N>1$. However, near $k_{10} h_{1}=3.5$, full reflection occurs in the case of a single barrier. The fashions in which the reflection coefficient varies with the parameters, and the appearance of the full reflection for $N=1$, are qualitatively the same as those found by Kaligatla et al. [13] for wave interaction with a single par- 
tial flexible porous barrier near a rigid wall in uniform water depth. Further, smaller wave reflection occurs in the case of flexible porous barriers as compared with rigid porous barriers. It is also seen that for $0.8<k_{10} h_{1}<1.8$, a single barrier can reduce the wave reflection notably. However, for $1.5<k_{10} h_{1}<3.8$, the wave reflection decreases with an increase in the number of barriers $N$. Thus, it is necessary to install multiple barriers if a single barrier produces little reduction on wave reflection. Further, Fig. 3(b) shows that double flexible porous barriers exhibit good performance in reducing wave reflection for all values of $k_{10} h_{1}$. Thus, for an effective breakwater, $N=2$ is a good choice.

\subsection{Effect of various wave and structural parameters}

In the following sections, the effects due to various wave and structural parameters on wave reflection, loading, and so on are discussed.

\subsubsection{Reflection coefficient versus non-dimensional wave number $k_{10} h_{1}$}

In Figs. 4(a) and 4(b), the reflection coefficient, $K_{r}$, is plotted as a function of the non-dimensional wave number, $k_{10} h_{1}$, for different values of the mooring line angle $\vartheta$ and porous-effect parameter $G$, respectively. From Fig. 4(a), it is seen that the minimum wave reflection is smaller with a larger mooring line angle $\vartheta$. On the other hand, the wave reflection is smaller for clamped-moored barriers $(\vartheta \neq 0)$ when compared with the case for clamped-free barriers $(\vartheta=0)$. This is due to the trapping of more waves between the barriers under the clamped-moored edge condition, as has been reported by Karmakar and Soares [18].

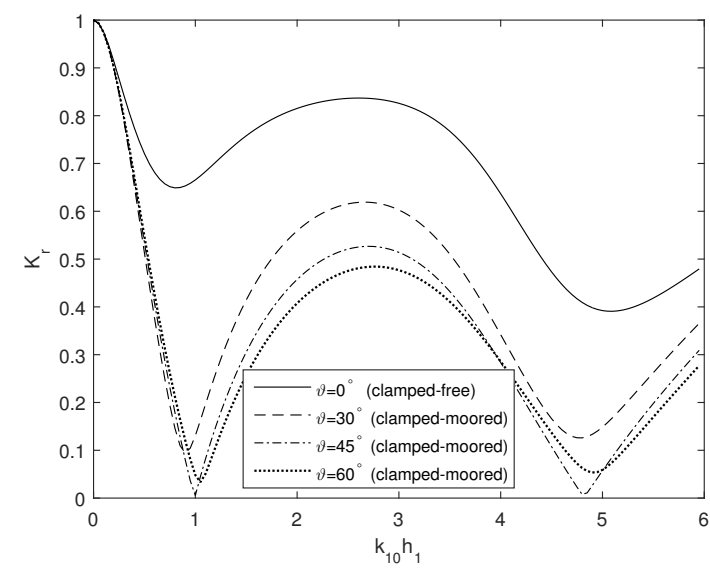

(a)

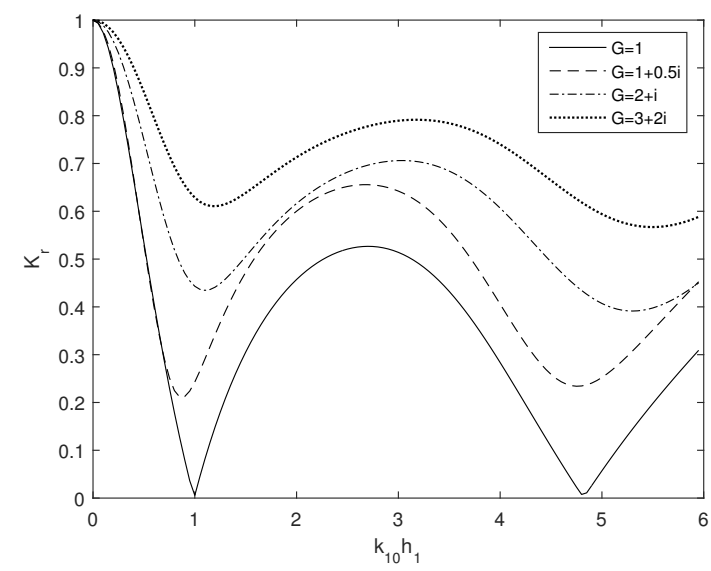

(b)

Figure 4: Effect of the (a) mooring line angle $\vartheta$ with $G=1$, and (b) porous-effect parameter $G$ with $\vartheta=45^{\circ}$, on the reflection coefficients $K_{r}$ as a function of the non-dimensional incident wave number $k_{10} h_{1}$, for $N=2, \gamma=0.1, \beta=0$, $D / h_{1}=0.4, L / h_{1}=0.2, b / h_{2}=1$, and $h_{2} / h_{1}=0.5$.

Fig. 4(b) depicts that the reflection coefficient increases with an increase in the absolute value of the porous-effect parameter $G$. This results from a higher transmission of wave energy through the flexible porous barrier with an increase in the absolute value of $G$. Further, there is a right shift of the wave reflection curves as the absolute value of the porous-effect parameter $G$ increases. This may 
be due to a phase change of the reflected wave with a change in $G$. It may be noted that nearly zero reflection occurs for $N=2, \vartheta=45^{\circ}$ and $G=1$ at $k_{10} h_{1}=1,4.9$. The nearly zero minimum reflection is referred to as wave trapping by flexible porous barriers near a rigid wall, as has been discussed by Yip et al. [11] for a single barrier.

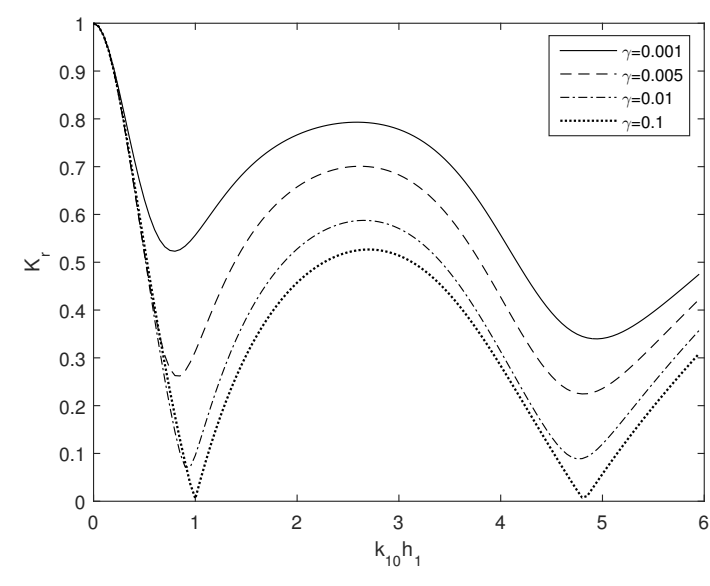

(a)

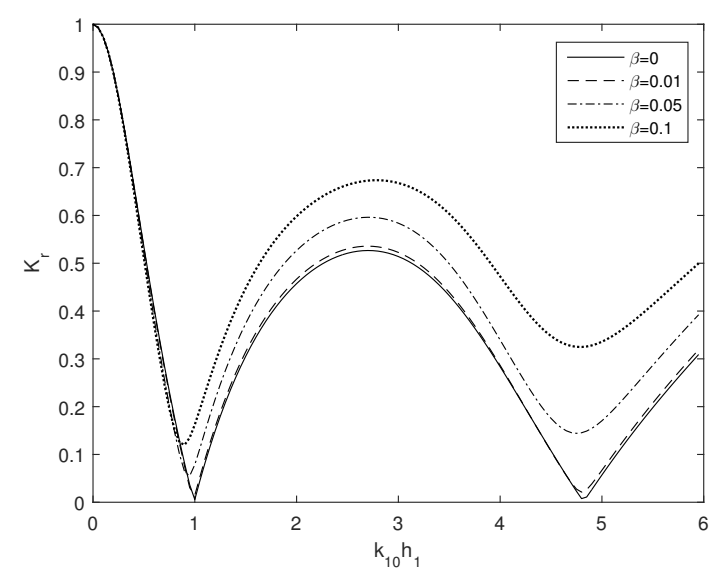

(b)

Figure 5: Effect of the (a) flexural rigidity parameter $\gamma$ with $\beta=0$, and (b) compressive force parameter $\beta$ with $\gamma=0.1$, on the reflection coefficients $K_{r}$ as a function of the non-dimensional incident wave number $k_{10} h_{1}$, for $N=2, G=1$, $\vartheta=45^{\circ}, L_{2} / h_{1}=0.6, D / h_{1}=0.4, b / h_{2}=1$, and $h_{2} / h_{1}=0.5$.

In Figs. 5(a) and 5(b), the reflection coefficient, $K_{r}$, is plotted as a function of the non-dimensional wave number, $k_{10} h_{1}$, for different values of the non-dimensional flexural rigidity $\gamma$ and compressive force $\beta$, respectively. From Fig. 5(a), we see that the wave reflection decreases with an increase in the structural rigidity. The wave reflection attains a minimum which is due to the destructive interference of higher modes of vibration of the flexible barriers with incident waves. For larger values of the structural rigidity, the porous barriers behave more like rigid porous barriers, and the results presented in this figure correspond to the case of wave interaction with double rigid porous barriers in the presence of a sloping step. Fig. 5(b) shows that with a change in the compressive force, there is negligible change in the wave reflection for smaller values of $k_{10} h_{1}$. Further, the wave reflection increases with an increase in $\beta$.

\subsubsection{Reflection coefficient versus non-dimensional slope length $L / \lambda_{1}$}

In Figs. 6(a) and 6(b), the reflection coefficient, $K_{r}$, is plotted as a function of the non-dimensional slope length, $L / \lambda_{1}$, for different values of length of the barrier $b / h_{2}$ and bed profile parameter $\delta$, respectively. From these figures, it is seen that the oscillatory pattern of the reflection coefficient curves weakens with an increase in the slope length $L / \lambda_{1}$. Further, toward smaller values of the slope length, the oscillatory pattern of the wave reflection curves is larger in amplitude. This may be due to the fact that the slope angle becomes larger for smaller slope length which leads to a higher resonating pattern of the resultant waves in the confined zone. Fig. 6(a) shows that the wave reflection decreases with an increase in the barrier height $b / h_{2}$. This is a result of a higher dissipation of wave energy as the barrier height increases. On the other hand, because of the protrusion and depression of the bed profile, the reflection coefficient increases with an increase in the value of $\delta$, as shown in Fig. 6(b). 


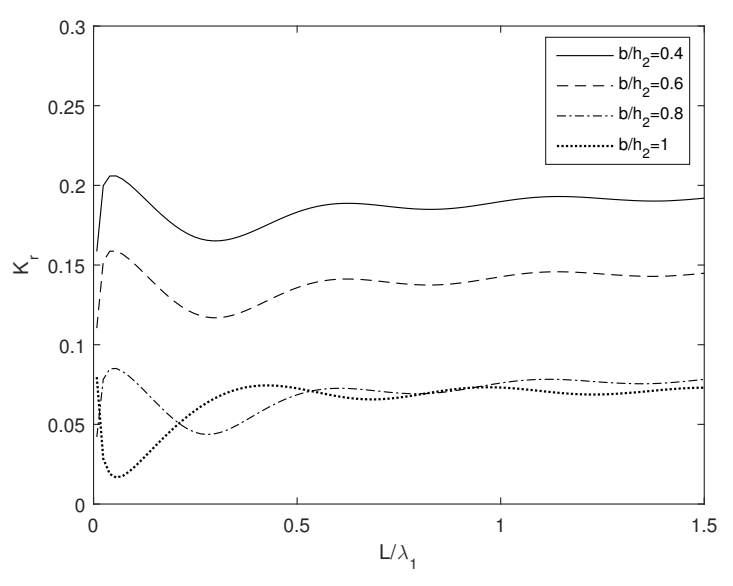

(a)

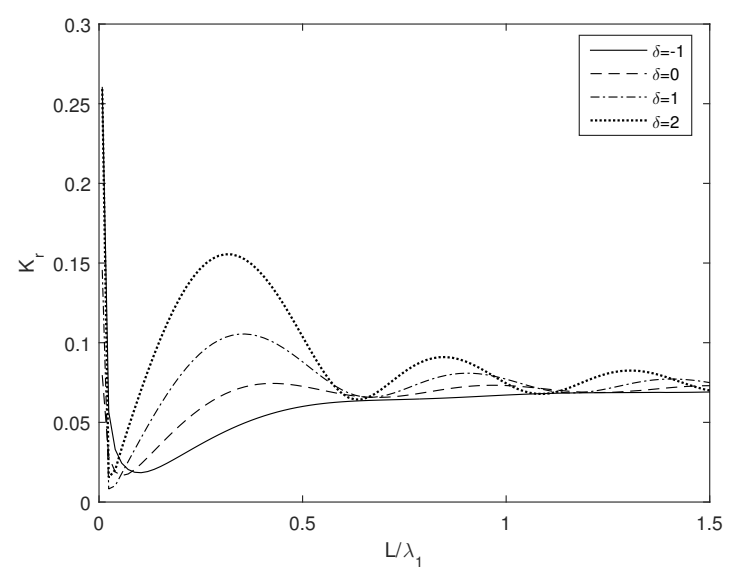

(b)

Figure 6: Effect of the (a) normalized length of barriers $b / h_{2}$ with $\delta=0$, (b) bed profile parameter $\delta$ with $b / h_{2}=1$, on the reflection coefficients $K_{r}$ as a function of the ratio of step length to incident wavelength $L / \lambda_{1}$, for $k_{10} h_{1}=1, N=2$, $h_{2} / h_{1}=0.5, \vartheta=45^{\circ}, D / B=0.4$, and $\theta=40^{\circ}$.

\subsubsection{Reflection coefficient versus normalized distance between the first barrier and the rigid wall $B / \lambda_{1}$}

In Figs. 7(a) and 7(b), the reflection coefficient, $K_{r}$, is plotted as a function of the normalized distance between the first barrier and the rigid wall, $B / \lambda_{1}$, for different numbers of barriers $N, h_{2} / h_{1}=$ $1,0.5$ and $\theta=0^{\circ}, 40^{\circ}$. From these figures, for $N=1$, it is found that the optimum wave reflection occurs for larger value of $B / \lambda_{1}$ with an increase in $\theta$. This is due to a change in the phase of the incident wave with an increase in $\theta$. The patterns of the wave reflection for $N=1$ and $\theta=0^{\circ}$ as shown in Fig. 7(a) agree well with the results of Yip et al. [11] in the case of a single barrier. It is also seen that full wave reflection occurs periodically with an increase in $B / \lambda_{1}$ in the case of a single barrier. However, full reflection does not occur in the case of multiple barriers. Further, for $N=4$, nearly zero reflection occurs at $B / \lambda_{1}=0.18$. On the other hand, the patterns of the wave reflection for $N=1,2, \theta=40^{\circ}$ and $h_{2} / h_{1}=0.5$ as shown in Fig. 7(b) are similar to those presented by Li et al. [4] in the case of wave interaction with double rigid porous barriers. This agreement with previous studies gives further support to the correctness of the present results. It may be noted that full and nearly zero reflection occur for $\theta=0^{\circ}$ in the presence of a single barrier and sloping step of height $h_{2} / h_{1}=0.5$ as shown in Fig. 7(b).

Also, it is seen that full reflection occurs when $B / \lambda_{1}$ approaches zero. For $N=1$, full reflection occurs periodically as $B / \lambda_{1}$ increases. However, full reflection does not occur for $N>1$. It is also found that full reflections occurs for smaller values of $B / \lambda_{1}$ for $h_{2} / h_{1}=0.5$ when compared with the counterparts for $h_{2} / h_{1}=1$. From these figures, it is seen that in the regions of $B / \lambda_{1}$ where minimum wave reflection occurs, a single barrier exhibits better performance. However, in the regions of $B / \lambda_{1}$ where maximum wave reflection occurs, the wave reflection decreases with an increase in the number of barriers. Thus, the number of barrier to be installed for reducing wave reflection depends on the values of $B / \lambda_{1}$. 


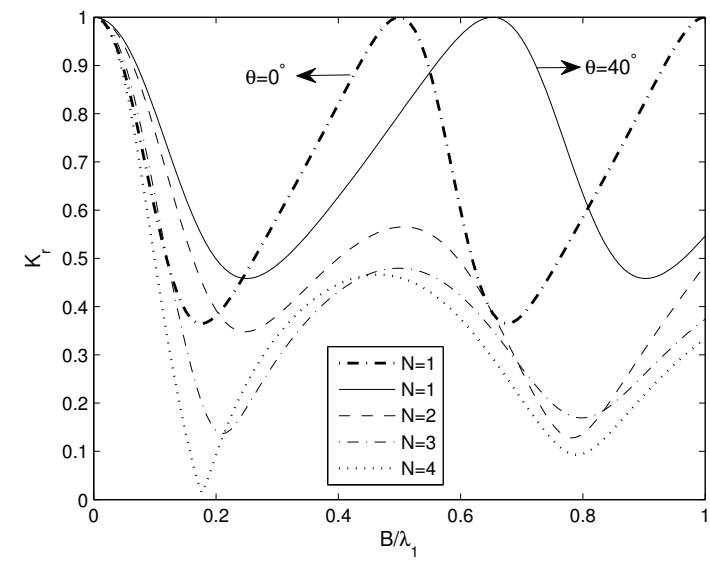

(a)

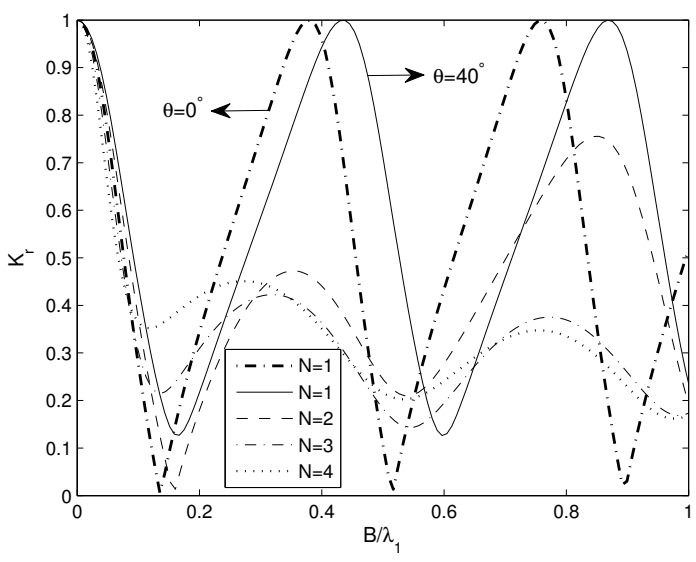

(b)

Figure 7: Effect of the number of barriers $N$ on the reflection coefficient $K_{r}$ as a function of the ratio of distance from wall of the first barrier to incident wavelength $B / \lambda_{1}$, with (a) $h_{2} / h_{1}=1$, and (b) $h_{2} / h_{1}=0.5$, for $k_{10} h_{1}=1, \gamma=0.1, \beta=0$, $\vartheta=45^{\circ}, L / h_{1}=0.2$, and $D / B=0.4$.

\subsubsection{Reflection coefficient versus angle of incidence $\theta$}

In Figs. 8(a) and 8(b), the reflection coefficients $K_{r}$ and the non-dimensional horizontal force $K_{w}$ are plotted as functions of the angle of incidence, $\theta$, for different values of the porous-effect parameter $G$, respectively. Fig. 8(a) shows that for $G=1$, minimum wave reflection occurs at a particular incident angle $\theta=40^{\circ}$. This particular angle of incidence is referred to as the critical angle, for which maximum wave trapping happens in the confined regions. Furthermore, the minimum reflection coefficient increases in magnitude with an increase in the absolute value of the porouseffect parameter $G$ as shown in Fig. 4(b). Fig. 8(b) reveals that the horizontal wave force exerted on the rigid wall $K_{w}$ increases with an increase in the absolute value of the porous-effect parameter $G$. This results from the fact that more waves will pass through the fine pores of the barriers with an increase in the absolute value of the porous-effect parameter, and thus a larger force will be exerted on the rigid wall.

\subsection{Non-dimensional horizontal wave force $K_{w}$ versus non-dimensional wave number $k_{10} h_{1}$}

In Figs. 9(a) and 9(b), the non-dimensional horizontal wave force acting on the rigid wall, $K_{w}$, is plotted as a function of the non-dimensional wave number $k_{10} h_{1}$ and the normalized distance between the first barrier and the rigid wall, $B / \lambda_{1}$, for different numbers of barriers $N$, respectively. From these figures, we can see that the horizontal force acting on the rigid wall $K_{w}$ decreases with an increase in the number of barriers $N$. This is expected since larger $N$ will lead to a higher rate of dissipation of wave energy by the porous barriers. Again, the number of barriers $N$ is one of the important factors in the breakwater design.

Further, the oscillatory pattern of the wave force curves decreases in amplitude with an increase in $N$. On the other hand, a comparison between Fig. 3(b) and Fig. 9(a), and between Fig. 7(b) and Fig. 9(b) reveals the values of $k_{10} h_{1}$ and $B / \lambda_{1}$ for which the wave reflection attains the maximum, while the wave force exerted on the rigid wall also reaches the maximum. Similar observation has been made by Behera et al. [12] for wave trapping by a partial flexible porous near a rigid wall. 


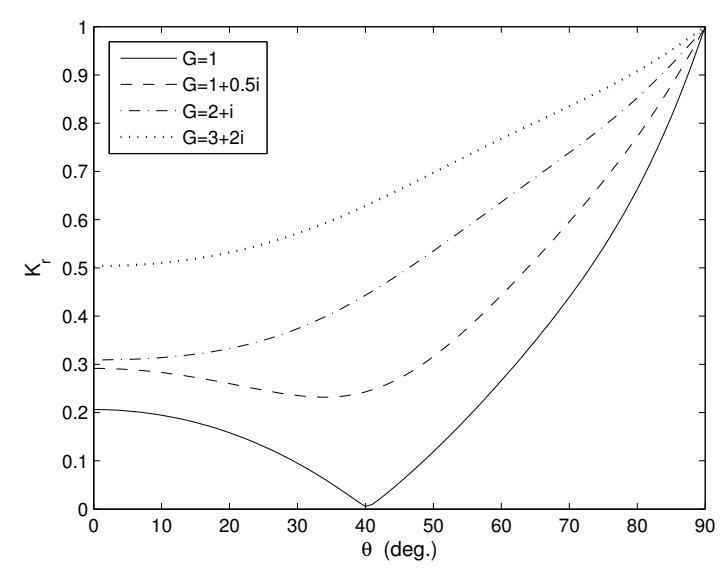

(a)

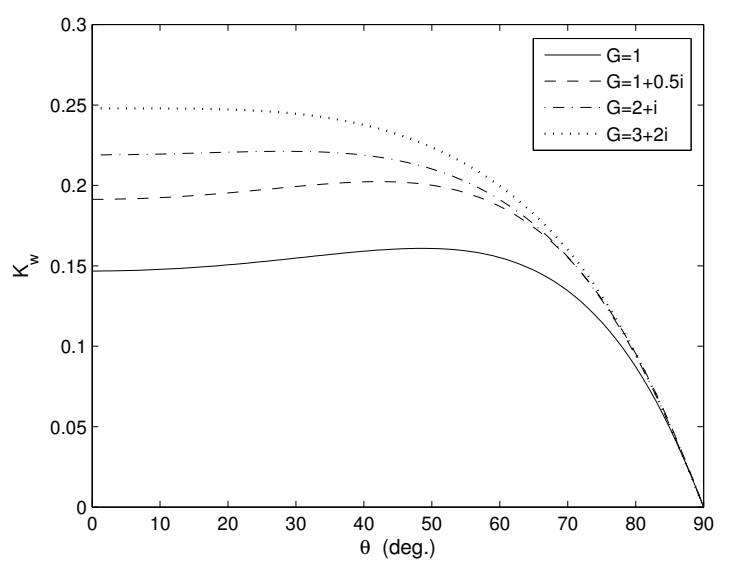

(b)

Figure 8: Effect of the porous-effect parameter $G$ on the (a) reflection coefficients $K_{r}$, and (b) non-dimensional horizontal force $K_{w}$, as functions of the wave incident angle $\theta$, for $k_{10} h_{1}=1, N=2, D / B=0.4, h_{2} / h_{1}=0.5, b / h_{2}=1, \gamma=0.1$, and $\vartheta=45^{\circ}$.

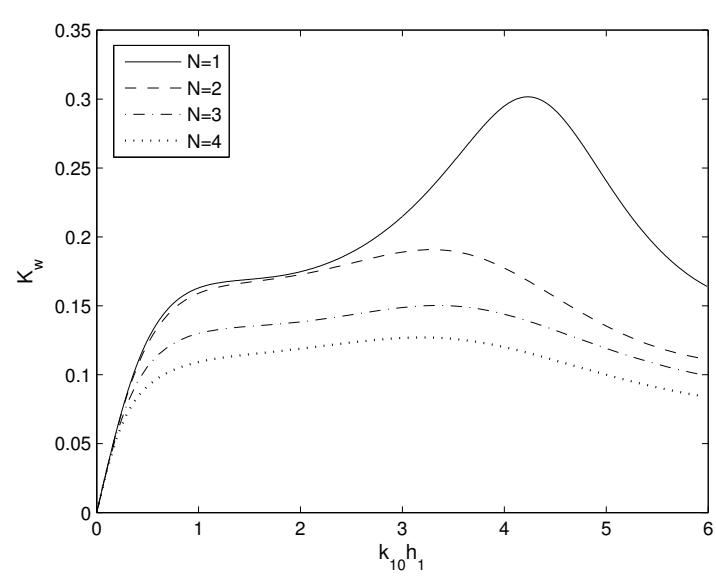

(a)

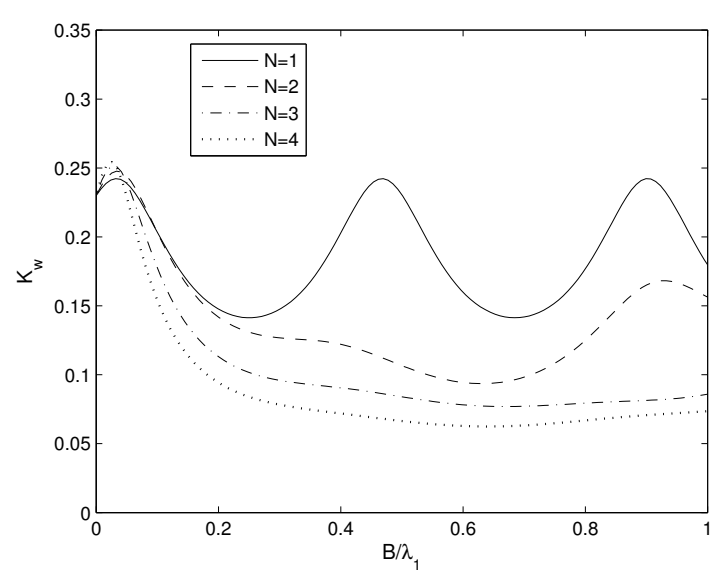

(b)

Figure 9: Effect of the number of barriers $N$ on the non-dimensional horizontal force $K_{w}$, as a function of the (a) nondimensional incident wave number $k_{10} h_{1}$, and (b) the ratio of distance from wall of the first barrier to incident wavelength $B / \lambda_{1}$, for $b / h_{2}=1, h_{2} / h_{1}=0.5, D / B=0.4, G=1, \vartheta=45^{\circ}, \gamma=0.1$, and $\theta=40^{\circ}$.

\subsection{Deflection of the barriers}

In Figs. 10(a) and 10(b), the deflection of the flexible porous barriers is plotted for different values of the mooring line angle, $\vartheta$, and the porous-effect parameter, $G$, in the case of double barriers. As expected, zero deflection occurs at the bottom, while the maximum deflection occurs near the free surface as all the barriers are clamped at the bottom and moored/free near the submerged end. The deflection of the first barrier is larger than that of the second barrier, as the the first barrier is impacted by higher wave force than the second. Fig. 10(a) shows that the barrier deflection decreases with an increase in the mooring angle. On the other hand, Fig. 10(b) reveals that the first barrier deflection decreases with an increase in the absolute value of the porous-effect parameter $G$. However, owing to the dissipation of wave energy by the first barrier, the deflection of the second barrier is not sensitive 


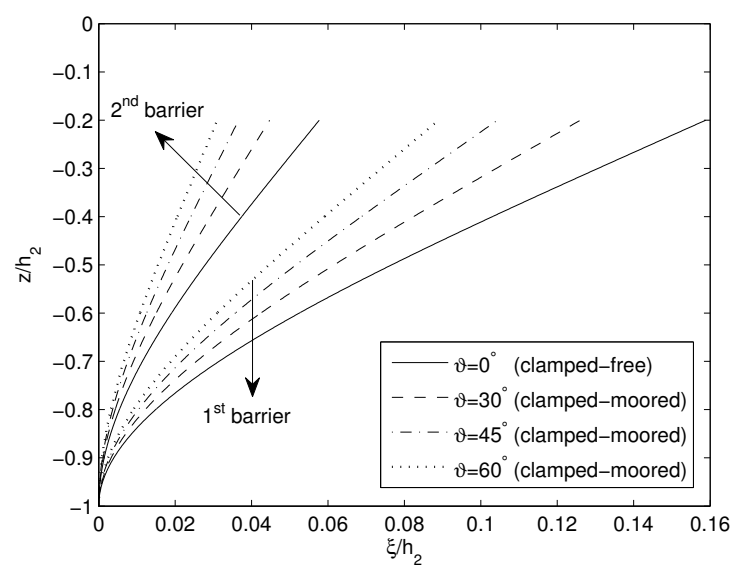

(a)

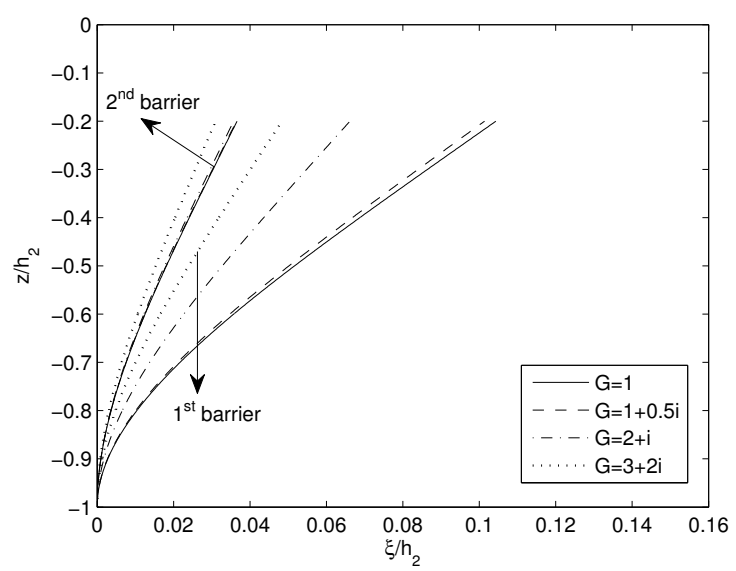

(b)

Figure 10: Effect of the (a) mooring line angle $\vartheta$ with $G=1$, (b) porous-effect parameter $G$ with $\vartheta=45^{\circ}$, on the normalized barrier deflection $\xi / h_{2}$, for $N=2, h_{2} / h_{1}=0.5, b / h_{2}=0.8, D / B=0.2, \gamma=0.1, \beta=0$, and $\theta=0^{\circ}$.

to the porous-effect parameter $G$.

\subsection{Free-surface elevations}

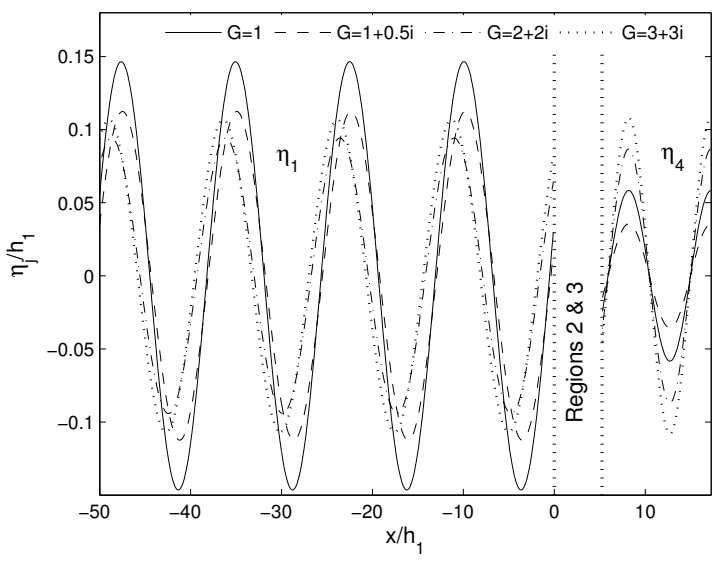

(a)

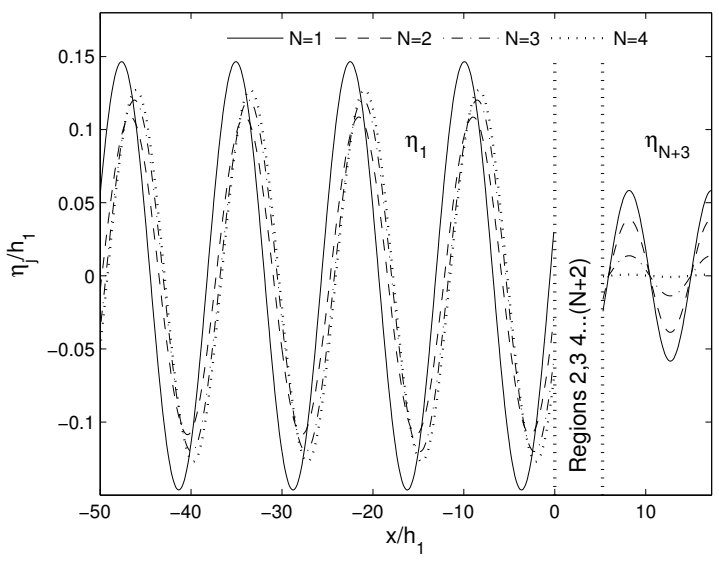

(b)

Figure 11: Effect of the (a) porous-effect parameter $G$ for single barrier with $L_{1} / h_{1}=5$, (b) different numbers of barriers with $G=1, L_{1} / h_{1}=0.5$ and $L_{2} / h_{1}=4.5 /(N-1)$ on the free-surface elevations $\eta_{j} / h_{1}$, for $h_{2} / h_{1}=0.5, b / h_{2}=1$, $L / h_{1}=0.2, \gamma=0.1, \beta=0$, and $\theta=0^{\circ}$.

In Figs. 11(a) and 11(b), the free-surface elevations $\eta_{j}$ for $j=1$ and $N+3$, i.e. in open water region 1 and rigid wall side regions $N+3$ are plotted for different values of (a) the porous-effect parameter, $G$, and (b) different numbers of barriers. Fig. 11(a) reveals that the amplitude of the free-surface elevation in the rigid wall region increases with an increase in the absolute value of the porous-effect parameter which is due to more wave energy passing through the barrier for larger $G$. Further, from Fig. 11(a), it is observed that in the presence of more barriers, there is a significant reduction on the amplitude of the free-surface elevation in the rigid wall side region. This is due to the wave trapping 
by adjacent porous barriers. Thus, for these wave and structural parameters, the confined zone can be made as a perfect tranquility zone.

\section{Conclusion}

The performance of multiple bottom-standing flexible porous barriers in the presence of a rigid vertical wall and a step-type bottom has been analyzed on the basis of linearized water wave theory. To determine the velocity potential in an undulated region, the modified mild-slope approximation is solved using the Runge-Kutta method while the eigenfunction expansion method is used for velocity potentials in the fluid domain having a uniform water depth. Further, the least-squares approximation method is used for finding the solutions in the presence of multiple partial flexible porous barriers. Numerical results are computed for the reflection coefficient, force on the rigid wall, deflection of the barriers, and free-surface elevations to reveal effects due to the associated wave and structural parameters. The present model is validated by comparing results with those available in the literature for the special case of wave interaction with single/double rigid porous barriers in the presence of a vertical step. The study reveals that a system of double flexible porous barriers is a good choice for an effective breakwater because it exhibits good performance in reducing wave reflection for all values of $k_{10} h_{1}$ (Fig. 3(b)). However, for reducing wave force on the rigid wall, multiple barriers as a breakwater plays an important role because it is found that the wave force exerted on the rigid wall significantly decreases with an increase in the number of barriers irrespective of the wave and structural parameters (Fig. 9). It is also observed that in the presence of a sloping step and single flexible porous barrier near a rigid wall, full and nearly zero wave reflection occur for certain wave and structural parameters (Fig. 3(b)). However, in the case of multiple barriers, full wave reflection does not occur but nearly zero wave reflection occurs for double barriers at a critical angle $\theta=40^{\circ}$ (Fig. 8(b)). These observations of nearly zero wave reflection will be of significance in the determination of maximum possible wave trapping by flexible porous barriers near a rigid wall. It is seen that under the clamped-moored edge condition, more wave is trapped between the barriers, and therefore wave reflection is smaller in this case as compared with clamped-free barriers (Fig. 4(a)). Unlike the case of uniform water depth, in the presence of step type bottoms, full reflection will happen periodically with an increase in the distance between the barrier and rigid wall for a single barrier (Fig. 7). In general, the reflection coefficient is lower for larger values of the mooring angle, structural rigidity and length of the barriers, while the reflection coefficient is higher for larger values of the porous-effect parameter, compressive force, protrusion and depression parameter of bed profile. The barrier deflection is found to be smaller for clamped-moored barriers as compared to clamped-free barriers. It is also observed that due to the dissipation of wave energy by the first barrier and wave trapping between the barriers, the barrier deflection is not sensitive to the porous-effect parameter. Further, it is seen that in the confined region (region between the last barrier and the rigid wall), with an increase in the number of barriers, the amplitude of the free-surface elevation decreases significantly. In particular, the amplitude of the free-surface elevation is very small in the presence of four barriers (Fig. 11(b)). This observation suggests that a calm region can be created on the lee side of the barriers. It is concluded that with the appropriate choice of wave and structural parameters, flexible porous barriers can act as an effective 
breakwater. Findings of the present study will guide engineers for the design of coastal structures to be constructed in the neighborhood of a sea wall or harbor wall.

\section{Conflict of Interest}

We have no conflict of interest.

\section{References}

[1] G. Jarlan, A perforated vertical wall breakwater, The Dock and Harbour Authority 41 (486) (1961) 394-398.

[2] K. D. Suh, W. S. Park, Wave reflection from perforated-wall caisson breakwaters, Coastal Engineering 26 (3) (1995) 177-193.

[3] T. Sahoo, M. Lee, A. Chwang, Trapping and generation of waves by vertical porous structures, Journal of engineering mechanics 126 (10) (2000) 1074-1082.

[4] Y. Li, G. Dong, H. Liu, D. Sun, The reflection of oblique incident waves by breakwaters with double-layered perforated wall, Coastal engineering 50 (1) (2003) 47-60.

[5] B. Teng, X. Zhang, D. Ning, Interaction of oblique waves with infinite number of perforated caissons, Ocean Engineering 31 (5) (2004) 615-632.

[6] Y. Liu, Y.-c. Li, B. Teng, The reflection of oblique waves by an infinite number of partially perforated caissons, Ocean engineering 34 (14) (2007) 1965-1976.

[7] Y. Liu, Y. Li, B. Teng, J. Jiang, B. Ma, Total horizontal and vertical forces of irregular waves on partially perforated caisson breakwaters, Coastal Engineering 55 (6) (2008) 537-552.

[8] Z. Huang, Y. Li, Y. Liu, Hydraulic performance and wave loadings of perforated/slotted coastal structures: a review, Ocean Engineering 38 (10) (2011) 1031-1053.

[9] H. Behera, R. Kaligatla, T. Sahoo, Wave trapping by porous barrier in the presence of step type bottom, Wave Motion 57 (2015) 219-230.

[10] Y. Liu, Y.-c. Li, B. Teng, Interaction between oblique waves and perforated caisson breakwaters with perforated partition walls, European Journal of Mechanics-B/Fluids 56 (2016) 143-155.

[11] T. L. Yip, T. Sahoo, A. T. Chwang, Trapping of surface waves by porous and flexible structures, Wave Motion 35 (1) (2002) 41-54.

[12] H. Behera, S. Mandal, T. Sahoo, Oblique wave trapping by porous and flexible structures in a two-layer fluid, Physics of Fluids (1994-present) 25 (11) (2013) 112110.

[13] R. Kaligatla, S. Koley, T. Sahoo, Trapping of surface gravity waves by a vertical flexible porous plate near a wall, Zeitschrift für angewandte Mathematik und Physik (2015) 1-26.

[14] M. H. Meylan, A flexible vertical sheet in waves, International Journal of Offshore and Polar Engineering 5 (02) (1995) 105-110.

[15] B. Mandal, A. Chakrabarti, Water wave scattering by barriers, Wit Pr/Computational Mechanics, 2000.

[16] W. K. Lee, E. Y. Lo, Surface-penetrating flexible membrane wave barriers of finite draft, Ocean engineering 29 (14) (2002) 1781-1804.

[17] D. Karmakar, J. Bhattacharjee, C. G. Soares, Scattering of gravity waves by multiple surface-piercing floating membrane, Applied Ocean Research 39 (2013) 40-52.

[18] D. Karmakar, C. G. Soares, Wave transformation due to multiple bottom-standing porous barriers, Ocean Engineering 80 (2014) 50-63.

[19] U. L. M. Hassan, M. H. Meylan, M. Peter, Water-wave scattering by submerged elastic plates, The Quarterly Journal of Mechanics and Applied Mathematics 62 (3) (2009) 321-344.

[20] M. H. Meylan, L. G. Bennetts, M. A. Peter, Water-wave scattering and energy dissipation by a floating porous elastic plate in three dimensions, Wave Motion.

[21] S. Das, T. Sahoo, Hydroelastic analysis of very large floating structure over viscoelastic bed, Meccanica (2016) $1-17$. 
[22] S. Koley, T. Sahoo, Oblique wave scattering by horizontal floating flexible porous membrane, Meccanica 52 (1-2) (2017) 125-138.

[23] P. G. Chamberlain, D. Porter, The modified mild-slope equation, J. Fluid Mech. 291 (1995) 393-407.

[24] D. Porter, D. Staziker, Extensions of the mild-slope equation, Journal of Fluid Mechanics 300 (1995) 367-382.

[25] X. Yu, A. T. Chwang, Wave motion through porous structures, Journal of engineering mechanics 120 (5) (1994) 989-1008.

[26] E. B. Magrab, Vibrations of elastic structural members, Sijthoff and Noordhoff, Alphen aan den Rijn, The Netherlands (1979).

[27] R. Porter, D. Porter, Water wave scattering by a step of arbitrary profile, J. Fluid Mech. 411 (2000) 131-164. 